\title{
Applying Contextual Constraints to Extract Symbolic Representation for Image Understanding
}

\author{
N. Semmar \\ Institut National des Sciences et Techniques Nucléaires \\ CE Saclay. 91191 Gif sur Yvette Cedex. France
}

\begin{abstract}
In this paper. we describe a parallel distributed processing model that learns to interpret the content of images using the surface features of their objects. These features are the relative positions of the basic objects in the image and their associated distinguishing attributes. The interpretation starts from the basic objects obtained in the morphological analysis phase and then applies a body of rules for the recursive recognition of the more complex objects contained in the image. The neural model assigns thematic roles to the image objects. disambiguates ambiguous objects. instantiate vague objects. and elaborates implied roles. The image are pre-segmented into a sequence of complex objects. Each object is processed in turn to update an evolving representation of the event described by the image. The model uses the information derived from each object to revise its ongoing interpretation of the image and to anticipate additional objects. The network learns to perform this interpretation through practise on processing example image/event pairs. The learning procedure allows the model to take a statistical approach to determine the concepts corresponding the missing units. The result of the interpretation process is the conceptual representation of the image in terms of the contained objects with their recognition degree.
\end{abstract}

\section{Introduction}

Images are very rich in semantics, and are subject to different interpretations according to the human perception or the application domain [1.2]. It is difficult to determine and represent the mutual relationships among the objects contained in an image because they form structures which vary greatly from image to image [3.t].

The automatic interpretation of image content is necessary to support the access to a database of images [5]. The goal of our research has been to develop a model that can learn to convert a simple image into a conceptual representation of the event that the image describes. Specifically, we have been concerned with the later stages of this process: the conversion of a sequence of image objects into a representation of the event. The event constitutes the symbolic representation of the image.

Section 2 presents the description of the image interpretation system. The accomplishments of the model are reported in Section 3. In section 4, we describe the limits of the model and further research efforts. 


\section{Description of the image interpretation system}

Extraction of concepts from an image means the conversion of the content of a simple image into a conceptual representation of the event that the image describes [6.7]. A number of problems make this process difficult. First, the objects of an image may be ambiguous. In the image containing a man with glasses. the object "man" is ambiguous, it could either refer to a teacher, bus driver or a doctor. How are the appropriate objects selected so that a single, coherent interpretation of the image is produced? A second problem is the complexity of assigning the correct thematic roles to the objects referred to in an image. In the image containing the objects: "man", "dog" and "leash", either the dog follows the man or the man follows the dog. then. if we do not know the spatial relationships between the objects man and clog we can not determine who is the agent and who is the patient. A third problem for processing content of images is that an image may leave some thematic constituents implicit which are nevertheless present in the event. For example in the image containing a teacher, a schoolgirl, and a blackboard. the teacher writes on the hlackhourd with chalk. Psychological evidence indicates that missing objects. when strongly related to the action, are inferred and added to the description of the event [8.9].

Our model of the interpretation process centres on viewing the process as a form of constraint satisfaction $[10,11,12]$. The surface features of an image. its particular objects and their importance provide a rich set of constraints on the image interpretation. Each feature constrains the interpretation in a number of respects. Conjunctions of features, such as objects importance order. provide additional constraints. Together, the constraints lead to a coherent interpretation of the image.

\subsection{Architecture of the neural network}

The image is presented to the model as a temporal sequence of objects. The information each of these image objects yields is immediately used as evidence to update the model's internal representation of the entire event [13.14]. From this representation, the model can produce, as output, a representation of the event. This event representation consists of a set of pairs. Each pair consists of a thematic role and the concept which fills that role. Together, the pairs describe the event.

\subsubsection{Producing the internal representation}

To process the objects of an image, we have adapted a neural network architecture with two hidden layers that uses the output of previous processing as input for the next iteration $[15,16]$ (Fig. 1). Each object is processed in turn to update the internal representation. An object, before being processed, it is first represented as a pattern of activation over the current object units. Activation from these units projects to the first hidden layer and combines with the activation from the internal representation created as the result of processing the previous object. The actual implementation of this arrangement is to copy the activation from the internal representation to the previous internal representation units, and allow activation to feed forward from there. Activation in the hidden layer, then, creates a new pattern of activation over the 
internal representation units. The internal representation, therefore, is not a superimposition of each object. Each new pattern in the image internal representation is computed through two layers of weights and represents the model's new best guess interpretation of the content of the image.

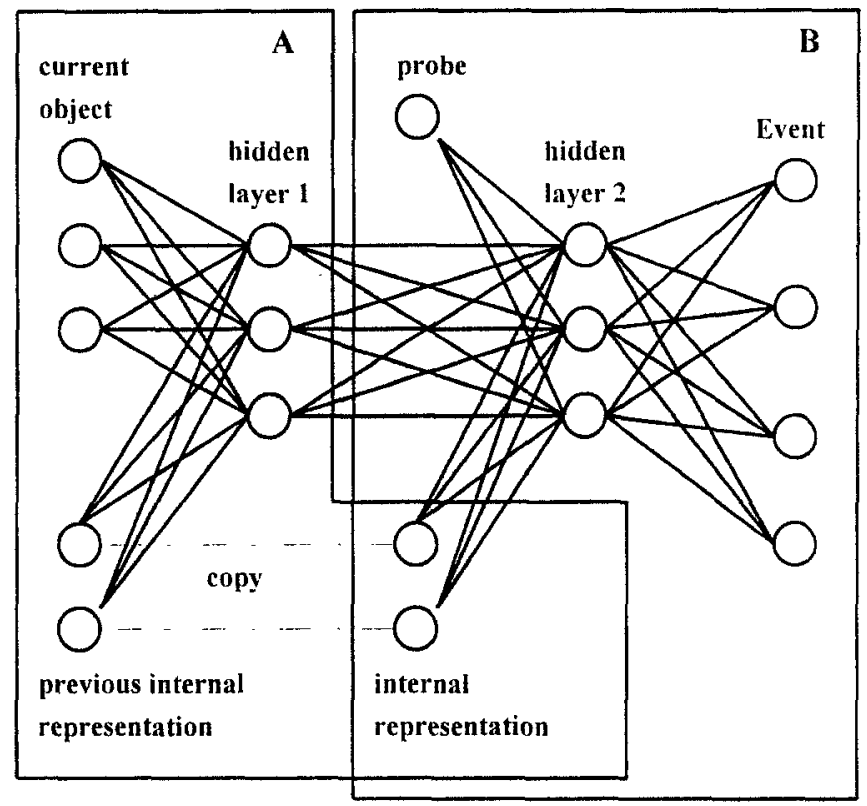

Fig. 1. The architecture of the neural network. The part $A$ processes the objects into the internal representation. and the part $B$ processes the internal representation into the output representation.

\subsubsection{Producing the output representation}

Our model makes the image internal representation a trainable hidden unit laver, which allows the network to create the primitives it needs to represent the content of the image efficiently. We used units that represent the conjunction of semantic features of the object with the semantic features of a concept. To encode an image. the patterns of activity produced for each object/concept conjunction were activated in a single pool of unit that contains every possible conjunction. Instead of having to represent every possible conjunction, only those conjunctions that are useful will be learned and added to the representation. Further, these primitives do not have to be conjunctions between the object and a concept. A hidden layer could learn to represent conjunctions between the concepts themselves or other combinations of information if they were useful for solving its task. Since a layer of hidden units cannot be trained by explicitly specifying its activation values, we decode the internal representation into an output layer. We use back propagation algorithın $[17,18]$ to train the hidden layer. The output layer represents the event as a set of thematic role and filler pairs. For example, the event described by an image of a man, an apple and a knife would be represented as the set $\{$ agent/man, action/peel, patient/apple, 
instrument/knife $\}$. The output layer can represent one role/filler pair at a time. To decode a particular role/filler pair, the internal representation is probed with half of the pair, either the role or the filler. Activation from the probe and the internal representation combine in the second hidden layer which in turn activates the entire role/filler pair in the output layer. The entire event can be decoded in this way by successively probing with each half of each pair.

When more than one concept can plausibly fill a role, we assume that correct response of the model is to activate each possible filler to a degree. The degree of activation of the units representing each filler corresponds to the filler's conditional probability of occurring in the given context. The network should learn weights to produce these activations through training. To achieve this goal, we employed an error measure in the learning procedure that converges on this goal:

$$
C=-\sum_{j}\left[T_{j} \log _{2}\left(A_{j}\right)+\left(I-T_{j}\right) \log _{2}\left(I-A_{j}\right)\right]
$$

Where $T_{j}$ is the target activation and $A_{j}$ is the output activation of unit $j$. The goal is to minimise the error measure. The minimum of $C$ occurs at a point in weight space where the activation value of each output unit equals the conditional probability that the unit should be on in the current context. When the network is probed with a particular filler of that role, several of the output units represent the occurrence of a particular filler of that role. When $C$ is at its minimum the unit's activation values represent the conditional probability of the occurrence of that filler, in that role, given the current situation. The minimum of $C$ is defined across the set of training examples the model is shown. Probing with the filler works similarly. The activation value of each role unit in the output layer represents the conditional probability of the probed filler playing that role in the current situation. In performing gradient descent in $C$. the network is searching for weights that allow it to match activations to these conditional probabilities.

\subsubsection{Training}

Training consists of trials in which the network is presented with an image and event it describes. The learner processes the image and compares the conceptual representation its interpretation mechanism produces to the conceptual representation it obtained from experiencing the event. Discrepancies are used as feedback for the interpretation mechanism. These image/event pairs were generated on-line for each training trial.

While images often include ambiguous objects, the events are always specific and complete: each event consists of a specific action and each thematic role related to this action is filled by some specific concept. Accordingly, each event occurs in particular location, and actions requiring an instrument always have a specific instrument.

During training, the objects of the image are presented to the model sequentially, one at a time. After the model has processed an object, the model is probed with each half of each role/filler pair for the entire event. The error produced on each probe is collected and propagated through the network. The weight changes from each image trial are added together and used to update the weights after every several trials. 


\subsection{Functionalities and performance of the model}

First. we will assess the model's ability to comprehend images generally. Then we will examine the model's ability to fulfil our specific processing goals, and we will examine the development of the model's performance across training trials. Finally. we will discuss the model's ability to generalise.

\subsubsection{Overall performance}

Correct processing was defined as activating the correct units more strongly than the incorrect units. After 10000 random image trials, the model began correctly processing the image in the corpus. A set of 100 test image/event pairs were generated randomly from the corpus. These image/event pairs were generated in the same way the training images were generated except that they were generated without regard to their frequency during training, so seldom practised pairs were as likely to appear in the test set as frequently practised pairs. Of these pairs. 45 were set aside for separate analysis because they were ambiguous: at least two different interpretations could be derived from each (e.g. A man with glasses). Of the remaining image event pairs. every image contained at least one vague or ambiguous object. yet each had only one interpretation. These unambiguous image/event pairs were tested by tirst allow ing the model to process all of the objects of the image. Then the model was probed with each half of each object that was mentioned in the image. The output produced in response to each probe was compared to the target output. For these unambiguous images. the cross-entropy. summed over constituents. averaged 3.9 per image. Another measure of performance is the number of times an output unit that should be on is less active than an output unit should be off. The idea behind this measure is that as long as the correct unit within any set, such as people or gender is the most active. it can win a competition with the other units in that set. Checking that all of the correct units are more active than any of the incorrect units is a quick. and conservative. Wa! of calculating this measure. An incorrect unit was more active in 14 out of the 2000 possible cases. or on $0.7 \%$ of the opportunities. The 14 errors were distributed over 8 of the 55 images. In 5 of the 8 images, the error involved the incorrect instantiation of the specific concept. or a feature of that concept, referred to by a vague object. Two other errors involved the incorrect activation of the concept representing a non vague object. In each case. the incorrect concept was similar to the correct concept. Therefore, errors were not random; they involved the misactivation of a similar concept or the misactivation of a feature of a similar concept. The errors in the remaining image involved the incorrect assignment of thematic roles in a image.

Additional practice, of course. improved the model's performance. Improvement is slow, however, because the images processed incorrectly are relatively rare. After a total of 20000 trials, the number of images having a cross-entropy higher than 15 dropped from 3 to 1 . The number of errors dropped from 14 to 11 . 


\subsubsection{Performance on specific tasks}

Our specific interest was to deyelop a processor that could correctly perform several important image interpretation tasks. Three specific images were drawn from the corpus to test each processing task. The categories and one example image for each are presented in Table 1. The parentheses denote the implicit, to be inferred. role.

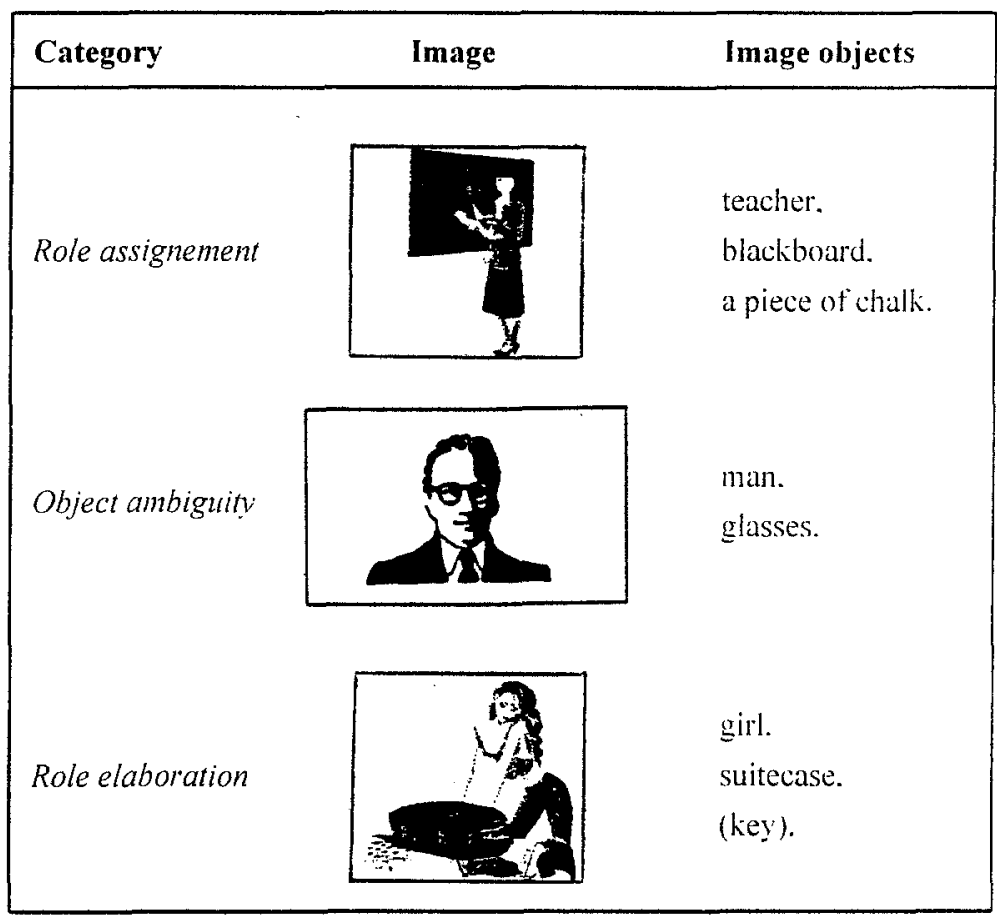

Table 1. Task categories

The first category involves role assignment. In the example containing a teccher. a blackboard and a piece of chalk, of the concepts referred to in the image. only the teacher can play the role of an agent of teaching. The network can therefore use that semantic information to assign teacher to the agent role. After each image was processed, the internal representation of the image was probed with the half of each role/filler pair. The network then had to complete the pair by filling in the correct thematic role. For each pair, in each image, the unit representing the correct role was the most active. Of course the semantic knowledge necessary to perform this task is never provided in the input or programmed into the network. Instead. it must be developed internally in the internal representation of the image as the network learns to process images.

Surface information does not have to be used in these cases; the semantic constraints suffice. In fact. if the surface location of the objects is removed from the input, the 
roles are still assigned correctly. Further, if the objects are presented in different orders. the activation values in the output are affected only slightly.

The remaining two categories involve the use of context to help specify the concepts referred to in an image. Images in the object ambiguity category contain one or more ambiguous objects. After processing an image, the network was probed with the role half of each role/filler pair. The output patterns for the fillers were then examined. For all pairs in each test image, the correct filler was the most active.

Desambiguation requires the competition and co-operation of constraints of both the object and its context. While the object itself cues two different interpretations. the context fits only one. In the image containing the objects "man" and "glasses". the object "man" is ambiguous. it could either refer to a teacher, hus driver or a cloctor. The context cues both teacher and doctor because the model has seen images involving both people with glasses. All the constraints supporting doctor combine. and together they win the competition for the interpretation of the image.

Depending upon the image, however, the context may only partially constrain the interpretation. Such is the case in the image containing a teacher and a sir. The object "girl" could refer to any of the people found in the corpus. Since. in the network's experience. in a classroom, we can find schoolboys and schoolgirls. the context constrains the interpretation of girl to be the schoolgirl. Consequently. the model can activate the female and girl features of the patient while leaving the unit representing the schoolgirl only partially active. The features adult and child are also partially and equally active because the teacher is an adult while the schoolgirl is a child. While schoolgirl is slightly more active in this example. neither is activated above 0.5 . In general. the model is capable of inferring as much information as the evidence permits: the more evidence, the more specific the inference.

Object disambiguation can be seen as one type of this general inference process. The only difference is that for ambiguous objects. both the general concept and the specific features differ between the alternatives. while for vague objects. the general concept is the same and only some of the specific features differ.

Finally, images in the role elaboration category test the model's ability to infer thematic roles not mentioned in the input image. For example, in the image containing the objects: "girl" and "suitcase", no instrument is mentioned. yet a ki'l can be inferred. For each test image, after the image was processed. the network was probed with the role half of the to-be-inferred role/filler pair. The correct filler was the most active in each case. For role elaboration. the context alone provides the constraints for making the inference. Extra roles that are very likely will be inferred strongly. When the roles are less likely, or could be filled by more than one concept. they are only weakly inferred.

As it stands, there is nothing to keep the network from generalising to infer extra roles for every image. even for events in which these roles make no sense. For instance, in the image containing a teacher and a blackboard (Fig. 2.), a stick should be inferred, yet the network infers a piece of chalk because of its association with the teacher. It appears that since the teacher uses a piece of chalk in many events about teaching, the network generalises to infer the piece of chalk as an instrument for this action. However. in events further removed from teaching. instruments are not inferred. For example, in the image containing a teacher and a rose. no instrument is 
activated. It appears, then, that generalisation of roles is affected by the degree of similarity between events. When events are distinct, roles do not generalise. and the model has no reason to activate any particular filler for a role.

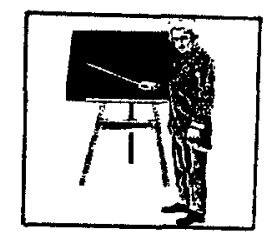

Fig. 2. An image with the objects: teacher, blackboard and stick.

\section{Accomplishments of the model}

The extraction of concepts from the image objects is based on conceptual (surface and semantic) constraints. The surface constraints are more difficult for the model to master then the semantic constraints even though we have provided explicit cues to the surface features, in the form of the surface location of the object. in the input. The model masters these constraints as they are exemplified in the set of training images.

The model infers unspecified objects roughly to the extent that they can be reliably predicted from the context. Here, we see very clearly that objects of an event description can be cued without being specifically designed by any object of the image. These inferences are graded to reflect the degree to which they are appropriate given the set of clues provided. The drawing of these inferences is also completely intrinsic to the basic interpretation process: no special separate inference processes must be spawned to make inferences, they simply occur implicitly as the objects of the image processed.

The model demonstrates the capacity to update its representation as each new object is encountered. As each object is encountered. the interpretation of all aspects of the event description is subject to change. If we revert to thinking in terms of meanings of concepts describing particular objects, both prior and subsequent context can influence the interpretation of each object. The ability to exploit subsequent context is an intrinsic part of the process of interpreting each new object. There is no backtracking, the representation of the image is simply updated to reflect the constraints imposed by each object as it is encountered.

While avoiding backtracking, the model also avoids the computational explosion of computing each possible interpretation of an image as it encounters ambiguous objects and thematic role assignments. When an ambiguous object is processed. it initially activates semantic units representing both concepts. The resulting pattern of activation is a combination of the semantic features of both concepts, so the bindings among the features of a concept are lost in the activation pattern. These bindings, however are preserved in the weights, and the model will settle into one interpretation of the object or the other. 
Finally the model is able to generalise the processing knowledge it has learned and apply it to new images. To perform this generalisation task. the model learns several types of information. First, it learns the concept referred to by each object in an image. Second, the model learns the order of the objects, which object comes before the main object and which after. This order is important to assigning the agent and patient. Third, the model learns the relevance of relationships among each object and the main object of the image. Fourth. the model learns to integrate the objects order and the information obtained from relationships to correctly assign the thematic roles.

\section{Conclusion}

The approach described in this article has been experimented on simple images. It will be important to analyse the limitation of the model on the complexity of the images. and the events that they describe [19]. Indeed. it is necessary to characterise the roles and fillers of images with respect to their super ordinate objects. The images are limited in complexity because of the limitations of the event representation. Only one filler can be assigned to a role in a particular image. Also. all the roles are assumed to belong to the image as a whole. Therefore. no embedded actions attached to single objects are possible. Similarly in complex events. there may be more than one actor. each performing an action in a different sub-event of the overall event or action. Representing these structures requires head/role/filler triples instead of simple role/filler pairs.

\section{References}

1. S. Zeki, S. Shipp: The functional Logic of Cortical Connections. In nature vol. 335, n6188. pp.311-317 (1988).

2. P.A. Carpenter. M.A. Just: Reading comprehension as the eyes see it. In M.A. Just and P.A. Carpenter, eds.. Cognitive Processes in Comprehension. Erlbaum, Hillsdale, NJ (1977).

3. C. Faloutsos. T. Sellis, N. Roussopoulos: Analysis of object oriented spatial access methods. In Proc. ACM SIGMOD International Conference. pp. 426439 (1987).

4. F. Rabitti, P. Savino: Automatic indexation to support content-based retrieval: In Information Processing \& Management Vol. 28. No. 5, pp. 547-567 (1992).

5. N. Semmar, C. Fluhr: A neural model for image content analysis. In Proceedings of The Third Turkish Symposium on ARTIFICIAL INTELLIGENCE \& NEURAL NETWORKS. Ankara METU (1994).

6. N. Semmar, C. Fluhr: Tool kits design for the internationalisation of multimedia courseware. In Tech. Report DELTA programme D2013 project MALIBU, Deliverable 12 WorkPackage 24(3) (1993).

7. N. Semmar, C.Fluhr: Utilisation d'une représentation hybride des bases de données documentaires pour la recherche d'informations. In Actes des journées d'études sur les Systèmes d'Information Elaborée, lle Rousse Corse (1993). 
8. V. Gudivada. V. Raghavan: A system for retrieving images by content. In Intelligent Multimedia Information Retrieval Systems and Management. Rockefeller University, New York (1994).

9. T. -Y. Hou: A content-based indexing technique using relative geometry features. In Storage and Retrieval for Image and Video Databases. SPIE. Vol. 1662 (1992).

10. J. Hertz, A. Krogh, R.G. Palmer: Introduction to the Theory of Neural Computation. Addison-Wesley (1986).

11. G.E. Hinton: Connectionnist Learning Procedures. In Artificial Intelligence vol. 40, n'1-3. pp.185-234 (1988).

12. G.E. Hinton: Learning distributed representations of concepts. In Proceedings of Eighth Annual Conference of the Cognitive Science Society, Amherst. MA (1986).

13. J.L. McClelland, D.E. Rumelhart: An interactive activation model of context effects in letter perception: Part 1. An account of basic findings. In Psychol. Rev.88, pp. 375-407 (1981).

14. M.L. Jordan: Attractor dynamics and parallelism in a connectionist sequential machine. In Proceedings Eighth Annual Conference of the Cognitive Science Society, Amherst, MA (1986).

15. D.E. Rumelhart. J.L. McClelland: Explorations in Parallel Distributed Processing, A Handbook of Models, Programs, and Exercises. MIT Press (1988).

16. D.L. Waltz. J.B. Pollack: Parallel Interpretation of Natural Language. In Proceedings of The International Conference of Fifth Generation Computer Systems, ICOT (1984).

17. D. E. Rumelhart. G. E. Hinton, R. J. Williams: Learning Representations by Back-Propagating Errors. In Nature vol. 323, nº188, pp.533-536 (1986).

18. D. E. Rumelhart, G.E. Hinton. R. J. Williams: Learning Internal Representations by Error Propagation. In Parallel Distributed ProcessingExplorations in the Microstructure of Cognition. MIT Press (1986).

19. C.F. Herot: Spatial management of data. In ACM Trans. Database Syst. 5. pp. 493-513 (1980). 